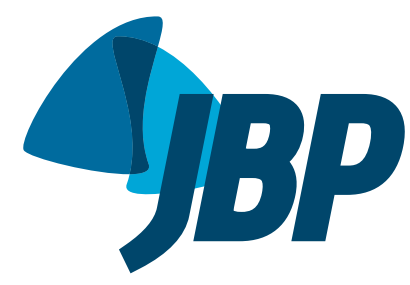

1. Divisão de Pneumologia, Instituto do Coração - InCor - Hospital das Clínicas, Faculdade de Medicina, Universidade de São Paulo, São Paulo (SP) Brasil.

a. (iD) http://orcid.org/0000-0003-2482-3127

b. (iD) http://orcid.org/0000-0003-0063-361X

c. (iD) http://orcid.org/0000-0001-7194-0479

d. (iD http://orcid.org/0000-0002-1618-8509

e. (iD) $h t t p: / / o r c i d . o r g / 0000-0002-9609-5117$

Submitted: 17 May 2017

Accepted: 15 October 2017

Study carried out in the Divisão de Pneumologia, Instituto do Coração - InCor - Hospital das Clínicas, Faculdade de

Medicina, Universidade de São Paulo, São Paulo (SP) Brasil.

\section{Characterization and outcomes of pulmonary alveolar proteinosis in Brazil: a case series}

\author{
Rodolfo Augusto Bacelar de Athayde ${ }^{1, a}$, Fábio Eiji Arimura ${ }^{1, b}$, \\ Ronaldo Adib Kairalla, ${ }^{1, c}$, Carlos Roberto Ribeiro Carvalho ${ }^{1, \mathrm{~d}}$, Bruno Guedes Baldi1,e
}

\begin{abstract}
Objective: Pulmonary alveolar proteinosis (PAP) is a rare disease, characterized by the alveolar accumulation of surfactant, which is composed of proteins and lipids. PAP is caused by a deficit of macrophage activity, for which the main treatment is whole-lung lavage (WLL). We report the experience at a referral center for PAP in Brazil. Methods: This was a retrospective study involving patients with PAP followed between 2002 and 2016. We analyzed information regarding clinical history, diagnostic methods, treatments, and outcomes, as well as data on lung function, survival, and complications. Results: We evaluated 12 patients (8 of whom were women). The mean age was 41 \pm 15 years. Most of the patients were diagnosed by means of BAL and transbronchial biopsy. The mean number of WLLs performed per patient was $2.8 \pm 2.5$. One third of the patients never underwent WLL. Four patients (33.3\%) had associated infections (cryptococcosis, in 2; nocardiosis, in 1; and tuberculosis, in 1), and 2 (16.6\%) died: 1 due to lepidic adenocarcinoma and 1 due to complications during anesthesia prior to WLL. When we compared baseline data with those obtained at the end of the follow-up period, there were no significant differences in the functional data, although there was a trend toward an increase in $\mathrm{SpO}_{2}$. The median follow-up period was 45 months (range, 1-184 months). The 5-year survival rate was $82 \%$. Conclusions: To our knowledge, this is the largest case series of patients with PAP ever conducted in Brazil. The survival rate was similar to that found at other centers. For symptomatic, hypoxemic patients, the treatment of choice is still WLL. Precautions should be taken in order to avoid complications, especially opportunistic infections.
\end{abstract}

Keywords: Pulmonary alveolar proteinosis; Bronchoalveolar lavage; Opportunistic infections.

\section{INTRODUCTION}

Pulmonary alveolar proteinosis (PAP) is a rare disease, characterized by the alveolar accumulation of surfactantwhich is composed of proteins and lipids-and caused by a deficit of macrophage activity. ${ }^{(1-3)}$ Epidemiological data show that the estimated prevalence of PAP is 0.37 cases per 100,000 population, most (approximately $90 \%$ ) of the cases having an autoimmune etiology. The mean age at diagnosis is in the fourth decade of life, PAP being more prevalent in men than in women and being associated with smoking. (3) The main symptoms are cough and dyspnea, and physical examination is usually unremarkable. ${ }^{(3,4)}$ The initial diagnosis of PAP is based on imaging findings (preferably HRCT findings) consistent with the disease, the most common CT finding being the crazy-paving pattern (septal thickening superimposed on areas of ground-glass attenuation typically bilateral and widespread). ${ }^{(5)}$ The diagnosis is then confirmed by the BAL fluid, which is typically milky in appearance. Although a diagnosis of PAP can be made without a surgical lung biopsy, the procedure is considered the gold standard for the diagnosis of PAP. ${ }^{(1,3,4)}$
On the basis of its etiology, PAP is classified as autoimmune PAP (formerly known as primary or idiopathic PAP), secondary PAP, or congenital PAP (also known as hereditary PAP). Autoimmune PAP is the most common of the three, being characterized by the presence of anti-GM-CSF antibodies. Secondary PAP is associated with hematologic diseases, neoplasms, toxic inhalations, and infections. Congenital PAP is the rarest form of PAP and usually occurs in children as a result of deficient surfactant production caused by mutations in the SFTPB gene, the SFTPC gene, the ABCA3 gene, or the NKX2-1 gene; changes in the GM-CSF receptor; or other mutations, such as GATA2 and telomerase complex mutations. ${ }^{(1-4)}$ Neutralizing anti-GM-CSF IgG antibodies can be found in the serum and BAL fluid of patients with autoimmune PAP. Autoantibodies neutralize alveolar macrophage activity and cause alveolar macrophage dysfunction, thus affecting surfactant catabolism and clearance from the distal air spaces. In cases of congenital PAP, defective surfactant clearance is primarily caused by mutations in genes encoding surfactant proteins or GM-CSF receptor chains, preventing GM-CSF from binding to its membrane

Correspondence to:

Rodolfo Augusto Bacelar de Athayde. Avenida Dr. Enéas de Carvalho Aguiar, 44, Bloco II, $5^{\circ}$ andar, sala 10, Cerqueira César, CEP 05403-900, São Paulo, SP Brasil.

Tel.: 5511 2661-5801. E-mail: rodolfobacelar1@hotmail.com

Financial support: None. 
receptor. ${ }^{(6)}$ In contrast, secondary PAP occurs in several diseases involving reduced alveolar macrophage numbers or function, including myelodysplastic syndromes, leukemias, and lymphomas, ${ }^{(1,7-10)}$ as well as solid tumors ${ }^{(11,12)}$ and infections (including tuberculosis, nocardiosis, and pneumocystosis), , $(1,4,6,8,10,13-17)$ together with environmental or occupational exposure to substances such as silica, aluminum, titanium, and certain fertilizers. $(1,3,4,6,8,13,18,19)$

The course of PAP varies widely among individuals, ranging from spontaneous resolution to rapid progression to respiratory failure. ${ }^{(3,5,20,21)}$ The treatment of PAP is variable, and, in cases of secondary PAP, the underlying cause should be treated. For patients with autoimmune PAP, available treatments include whole-lung lavage (WLL), GM-CSF replacement therapy, anti-CD20 monoclonal antibody (rituximab) use, and lung transplantation. ${ }^{(3,4)}$ During WLL, the initial effluent is typically milky and the supernatant is foamy because of the proteinaceous material filling the alveoli; as the procedure progresses, the effluent becomes clearer, ideally acquiring the same appearance as that of the instilled fluid. The 5-year survival rate ranges from $75 \%{ }^{(3)}$ to $95 \% .^{(1,4,13)}$ In 1991 , Lorenzi-Filho et al. ${ }^{(22)}$ reported a series of cases of patients who had been followed between 1983 and 1989 and in whom the diagnosis of PAP had been confirmed by biopsy, 3 patients having shown clinical and radiological improvement after WLL and 1 patient having achieved spontaneous remission. Below, we report the experience at a referral center for PAP in Brazil.

\section{METHODS}

This was a retrospective study involving PAP patients followed at our institution between 2002 and 2016, data being collected by reviewing patient medical records. We analyzed information regarding demographic characteristics, clinical history, diagnostic methods, treatments, and outcomes (including survival and complications).

Spirometry was performed with a calibrated pneumotachograph (Koko ${ }^{\circledR} \mathrm{PFT}$; nSpire Health Inc., Longmont, CO, USA). We collected data on the following variables: FVC, $\mathrm{FEV}_{1}$, and $\mathrm{FEV}_{1} / \mathrm{FVC}$. The predicted

Table 1. Clinical and demographic characteristics of the study sample $(\mathrm{N}=12)$. ${ }^{\mathrm{a}}$

\begin{tabular}{lc}
\multicolumn{1}{c}{ Variable } & Result \\
Female gender & $8(66.7)$ \\
Age, years & $41 \pm 15$ \\
Dyspnea at diagnosis & $12(100)$ \\
Smokers (current or former) & $2(16.6)$ \\
$\begin{array}{l}\text { Exposure } \\
\text { Birds }\end{array}$ & $3(25.0)$ \\
$\quad$ Mold & $1(8.3)$ \\
Diagnosis & \\
BAL & $9(75.0)$ \\
$\quad$ Open lung biopsy & $3(25.0)$ \\
\hline
\end{tabular}

aalues expressed as $\mathrm{n}(\%)$ or mean \pm SD. values were derived from the reference values for the Brazilian population. ${ }^{(23,24)}$ We also collected data on room-air pulse oximetry. We included no data on DLCO, because they were not consistently available for all patients at the time of the study. Given that arterial blood gas data were available for most of the patients who had undergone WLL, we evaluated pre- and post-WLL $\mathrm{PaO}_{2}$ values and alveolar-arterial oxygen gradients.

Continuous variables with normal distribution were expressed as mean and standard deviation, whereas those with non-normal distribution were expressed as median and interquartile range. Categorical variables were expressed as proportions. A paired t-test was used in order to compare functional data at diagnosis with the latest available functional data. Values of $p<0.05$ were considered significant. Survival was estimated by the Kaplan-Meier method. Data were analyzed with the program SigmaStat, version 3.5 (Systat Software, Inc., San Jose, CA, USA).

\section{RESULTS}

A total of 12 patients were included in the study. Their demographic and clinical characteristics are presented in Table 1 . Most $(66.7 \%)$ of the patients were female, and the mean age was $41 \pm 15$ years. Two patients were over 60 years of age. Most (75\%) of the patients had been diagnosed with PAP by BAL and transbronchial biopsy. A body mass index $>25$ $\mathrm{kg} / \mathrm{m}^{2}$ was a common finding, 4 patients being neither overweight nor obese. Despite having PAP, 1 young patient was a habitual cyclist. Treatments and primary outcomes are shown in Table 2. Pre- and post-WLL HRCT scans of the chest of 1 of the 12 patients in our series are shown in Figure 1, and the fluid collected during one of the WLL procedures performed is shown in Figure 2. One third of the patients never underwent WLL and remained clinically stable. In

Table 2. Treatments, major complications, and outcomes $(\mathrm{N}=12){ }^{\mathrm{a}}$

\begin{tabular}{lc}
\multicolumn{1}{c}{ Variable } & Result \\
WLL & \\
Number of patients undergoing WLL (n, \%) & $8(66.7)$ \\
Number of procedures per patient & $2.8 \pm 2.5$ \\
Other forms of treatment & \\
GM-CSF replacement therapy & $2(16.6)$ \\
Anti-CD20 monoclonal antibody & $2(16.6)$ \\
(rituximab) & \\
Neoplasm & $1(8.3)$ \\
Infection & $4(33.3)$ \\
Tuberculosis & $1(8.3)$ \\
Nocardiosis & $1(8.3)$ \\
Cryptococcosis & $2(16.6)$ \\
Death & $2(16.6)$ \\
Neoplasm & $1(8.3)$ \\
WLL complications & $1(8.3)$ \\
\hline
\end{tabular}

WLL: whole-lung lavage. aValues expressed as $\mathrm{n}(\%)$ or mean $\pm \mathrm{SD}$. 
contrast, 8 patients underwent WLL multiple times (mean number of procedures per patient, $2.8 \pm 2.5$; highest number of procedures per patient, 7 ; lowest number of procedures per patient, 2). The longest interval between 2 WLL procedures was 7 years, the mean interval between procedures being 1.9 years. With regard to other forms of treatment, 2 patients received GM-CSF replacement therapy, which resulted in improvement for 4 months in 1 , whereas another 2 received a single course of treatment with rituximab. Of the 12 patients studied, 4 (33\%) had opportunistic infections-cryptococcosis, in 2; nocardiosis, in 1; and tuberculosis, in $1-$ none of which were related to the treatments received. During the follow-up period, 3 patients reported exposure to birds and 1 patient reported exposure to mold. Of the 12 patients studied, 2 died: 1 of lepidic adenocarcinoma and 1 of pre-WLL complications (cardiopulmonary arrest secondary to hypoxemia during induction of anesthesia). Table 3

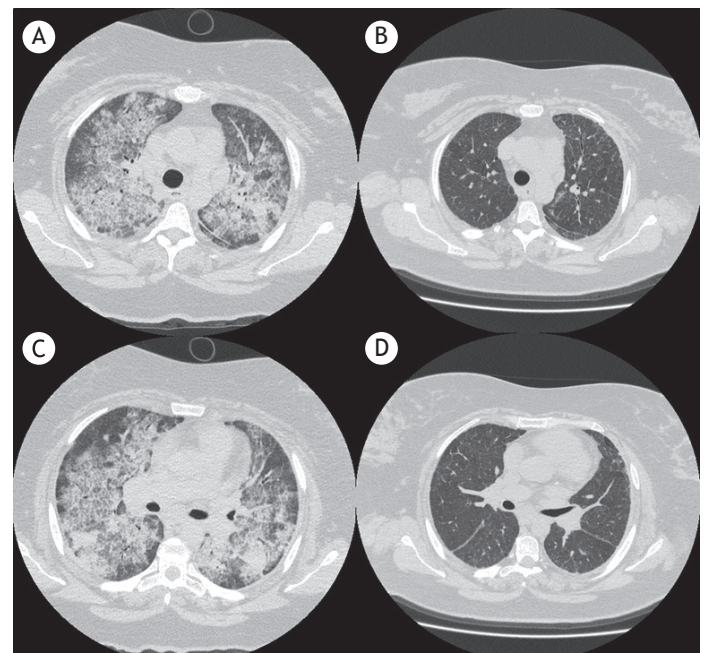

Figure 1. CT scans of the chest of a patient with pulmonary alveolar proteinosis, showing diffuse ground-glass opacities and interlobular septal thickening (the crazy-paving pattern). In $A$ and $C$, chest $C T$ scans performed before whole-lung lavage. In $B$ and $D$, chest CT scans performed after the procedure, showing improvement.

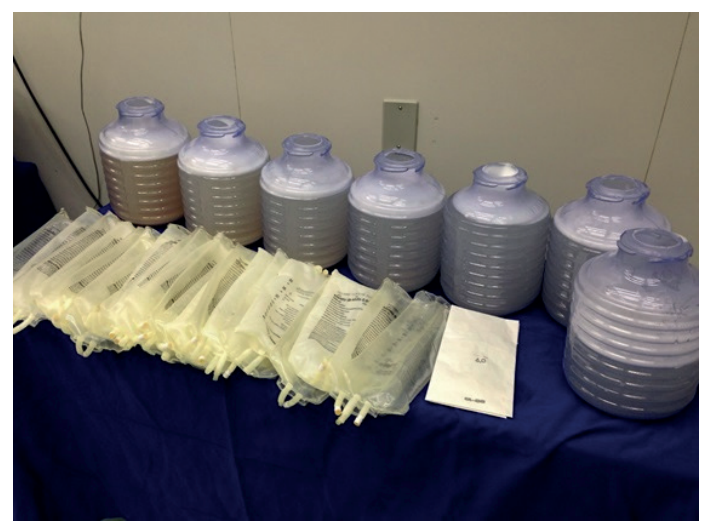

Figure 2. Flasks containing alveolar lavage fluid from a patient with pulmonary alveolar proteinosis. Note that the fluid became progressively less turbid (from left to right). shows data on lung function and $\mathrm{SpO}_{2}$ at diagnosis, as well as the latest available data (for the period from diagnosis to death or the last visit). No significant differences were found between baseline functional data and the latest available functional data, although there was a trend toward an increase in $\mathrm{SpO}_{2}$. Pre- and post-WLL arterial blood gas data were available for 5 patients. Mean $\mathrm{PaO}_{2}$ values increased from $48 \pm 10$ $\mathrm{mmHg}$ to $69 \pm 9 \mathrm{mmHg}(\mathrm{p}=0.01)$, whereas mean alveolar-arterial oxygen gradients decreased from 47 $\pm 9 \mathrm{mmHg}$ to $23 \pm 5 \mathrm{mmHg}(p=0.03)$. The median follow-up period (from diagnosis to death or the last evaluation) was 45 months (range, 1-184 months). The 1-, 3-, and 5-year survival rates were 91\%, 82\%, and $82 \%$, respectively (Figure 3 ).

\section{DISCUSSION}

Despite controversy, WLL remains the treatment of choice for PAP. The procedure is aimed at removing the surfactant material deposited in the alveoli as a result of macrophage dysfunction, thereby improving gas diffusion. More than $60 \%$ of patients undergoing WLL have been shown to respond well to two procedures per lung. Few patients require more than six WLL cycles, and less than $10 \%$ of patients do not respond to WLL. ${ }^{(25)}$ Asymptomatic or oligosymptomatic patients with normal or near-normal lung function and without hypoxemia

Table 3. Lung function and $\mathrm{SpO}_{2}$ : baseline data and latest available data for the $2002-2016$ period $(n=10)$.

\begin{tabular}{lccc}
\multicolumn{1}{c}{ Variable } & $\begin{array}{c}\text { Baseline } \\
\text { data }\end{array}$ & $\begin{array}{c}\text { Latest } \\
\text { available } \\
\text { data }\end{array}$ & $\mathbf{p}$ \\
$\mathrm{FEV}_{1}, \mathrm{~L}$ & $2.11 \pm 0.61$ & $2.16 \pm 0.43$ & 0.66 \\
$\mathrm{FEV}_{1}, \%$ predicted & $76 \pm 20$ & $77 \pm 15$ & 0.89 \\
FVC, L & $2.47 \pm 0.74$ & $2.62 \pm 0.54$ & 0.30 \\
FVC, \% predicted & $75 \pm 20$ & $78 \pm 15$ & 0.55 \\
$\mathrm{FEV}_{1} / \mathrm{FVC}$ & $0.85 \pm 0.05$ & $0.82 \pm 0.03$ & 0.18 \\
$\mathrm{SpO}_{2}, \%$ & $87 \pm 12$ & $94 \pm 7$ & 0.08 \\
\hline
\end{tabular}

avalues expressed as mean \pm SD.

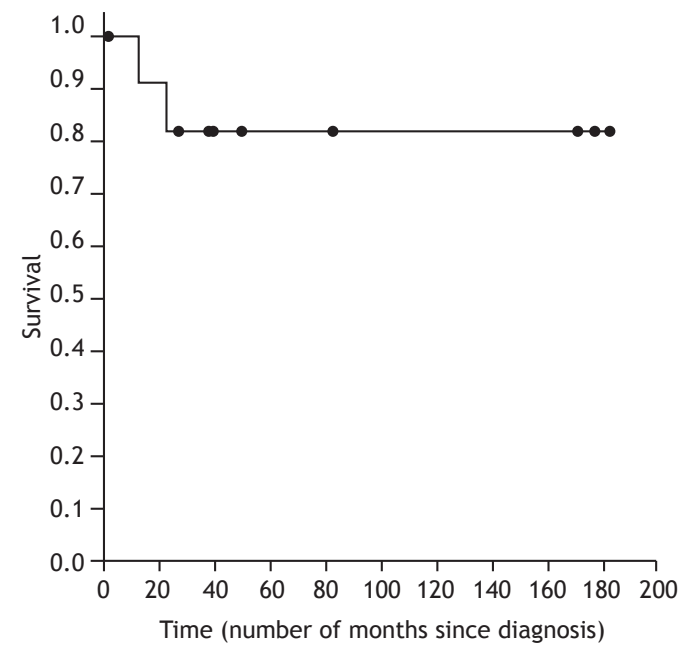

Figure 3. Kaplan-Meier survival curve. 
require periodic evaluation rather than immediate treatment. Symptomatic patients with moderate to severe lung function impairment, hypoxemia, or both can undergo WLL; although there is no consensus regarding which PAP patients should undergo WLL, those with moderate or severe dyspnea at rest or dyspnea on exertion and $\mathrm{a} \mathrm{PaO}_{2}$ of $<60 \mathrm{mmHg}$ at rest or an $\mathrm{SpO}_{2}$ of $<90 \%$ during exercise testing are candidates for the procedure. ${ }^{(2,6)}$ WLL is performed under general anesthesia, with the use of a double-lumen endotracheal tube and single-lung ventilation. Large saline volumes (of $10-15 \mathrm{~L}$ or more) are required, aliquots of $150-200 \mathrm{~mL}$ being infused. The initial returns are typically turbid, and the lavage should continue until the effluent becomes clear. A repeat WLL might

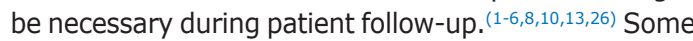
of our patients never underwent WLL and remained clinically stable, whereas others underwent multiple WLL procedures, findings that are consistent with the literature. In specific cases, segmental lung lavage is warranted. ${ }^{(1,4)}$ If the symptoms recur, if there is no response to WLL, or if WLL is contraindicated, inhaled or subcutaneous GM-CSF is an option. Another option is to use rituximab, an anti-CD20 monoclonal antibody. Both options have shown promising results. In our case series, 2 patients received GM-CSF replacement therapy. Of those, 1 remained clinically stable for 4 months but required a repeat WLL during clinical follow-up. Another 2 patients received a single loading dose of rituximab, a medication that is not widely available at our institution. Neither patient responded satisfactorily to treatment, possibly because the medication was used for a short period of time. Lung transplantation is an option for patients with advanced PAP. (1,3,4) $^{-1}$

Pulmonary function tests and exercise capacity tests play an important role in follow-up and therapeutic decisions. Although spirometry typically shows a restrictive pattern, results are normal in 10-30\% of cases. Smokers can present with an obstructive pattern. The most common and significant changes are hypoxemia and reduced DLCO. ${ }^{(1)}$ According to Borie et al., ${ }^{(1)}$ WLL results in symptomatic, radiological, and functional improvement in $85 \%$ of patients (mean improvement in $\mathrm{FEV}_{1}, 0.26 \mathrm{~L}$; mean improvement in FVC, $0.5 \mathrm{~L}$; mean improvement in DLCO, $4.4 \mathrm{~mL} / \mathrm{mmHg} /$ min; and mean improvement in $\mathrm{PaO}_{2}, 20 \mathrm{mmHg}$ ). We found no significant changes in lung function when we compared the data obtained at baseline with those obtained during the last visit. Although baseline values were at the lower limits of normal, they did not worsen over time. However, there was a trend toward an increase in $\mathrm{SpO}_{2}$. Arterial blood gas data were available for 5 patients. There was an increase in $\mathrm{PaO}_{2}$ and a decrease in the alveolar-arterial oxygen gradient after WLL, findings that confirm the impact of WLL on gas exchange.

Two patients died: 1 of lepidic adenocarcinoma and 1 of pre-WLL complications (cardiopulmonary arrest secondary to hypoxemia during induction of anesthesia), the latter patient having presented with a difficult airway. However, fatal complications are rare. ${ }^{(4,10)}$ Other possible complications include hydropneumothorax, pleural collections, endotracheal granuloma, stenosis due to multiple procedures, and surgical emphysema, ${ }^{(1,3,4,8,10)}$ none of which were observed in any of our patients. Of the 12 patients in our series, 1 was lost to follow-up. She was followed at our institution for 1 month only and was lost to follow-up because she resided in another state. The mean 5 -year survival rate was $82 \%$, which is similar to those found in other studies (i.e., 75-95\%). (1,3,4,13) $^{2}$

Precautions should be taken in order to avoid opportunistic infections in patients with PAP; macrophage deficiency, changes in surfactant proteins, and intra-alveolar accumulation of surfactant components result in a favorable environment for the growth of microorganisms. ${ }^{(3,4,8,10)}$ Major pathogens include Nocardia spp., Pneumocystis spp., Acinetobacter spp., Aspergillus spp., and Cladosporium spp., as well as Mycobacterium tuberculosis and other mycobacteria. ${ }^{(3,4,8,10)}$ Of the 12 patients in our series, 4 had opportunistic infections-cryptococcosis, in 2; nocardiosis, in 1; and tuberculosis, in 1-none of which were related to the treatments received. The infections were identified during the follow-up period, and all 4 patients responded well to pathogen-specific treatment.

Neoplastic diseases can cause secondary PAP, which is often associated with hematologic malignancies. ${ }^{(10,12)}$ There are few reported cases of PAP associated with solid organ cancer, PAP having been reported to occur prior to or coincidentally with lung neoplasms-including squamous cell lung carcinoma, adenocarcinoma, mesothelioma, glioblastoma, and metastatic melanoma-or breast cancer. ${ }^{(10,12)}$ With regard to autoimmunity, only 1 patient tested positive for ANF, rheumatoid factor, and anti-Ro antibodies. Although determination of serum levels of autoantibodies to GM-CSF is a simple and rapid test for autoimmune PAP, the sensitivity and specificity of which are nearly $100 \%$, it is not widely available. In addition, normal individuals and those with hematologic malignancies, particularly acute myeloid leukemia, can present with low titers for isotypes IgA, IgG, and IgM. The test is performed by ELISA (which is the gold standard method) and cell culture. A concentration $>19 \mathrm{mg} /$ $\mathrm{mL}$ is specific for autoimmune PAP, and values of $<10$ $\mathrm{mg} / \mathrm{mL}$ have a negative predictive value. No specific anti-GM-CSF autoantibody testing was performed in our sample. ${ }^{(1-3)}$

Occupational exposure (to silica, cellulose fibers, combustion products of plastics, aluminum, and titanium oxide) is known to contribute to the development of secondary $\operatorname{PAP}^{(3,4,8,18)}$; however, none of the individuals in our sample had a consistent history of exposure. Three patients (25\%) reported exposure to birds, and 1 patient reported exposure to mold. One of the patients who reported exposure to birds was a 47-year-old female patient who had quit smoking 1 year before symptom onset (with a smoking history of 
30 pack-years). She was obese (her body mass index was $35.6 \mathrm{~kg} / \mathrm{m}^{2}$ ), had had opportunistic nocardiosis, had undergone 7 WLL procedures (the longest interval between procedures being 6 months), and had received GM-CSF replacement therapy, having shown no clinical response. Clinical and functional improvement was achieved after the patient was separated from her pet bird (a cockatiel), which she had failed to mention. At this writing, no other intervention had been required, the patient having achieved remission 2 years prior. Her chest CT scans are shown in Figure 1, and the WLL fluid is shown in Figure 2. We found no studies examining this issue or reporting findings related to it. The association between PAP and smoking is also of note. Epidemiological data from other studies show that approximately $75 \%$ of patients have a history of smoking. ${ }^{(3,4)}$ However, only $16 \%$ of the patients in our sample were smokers.

In 1991, Lorenzi-Filho et al. published a case series of 4 patients who were in the 27- to 52-year age bracket and who were followed between 1983 and 1989 in Brazil. (22) In all 4 patients, the diagnosis of PAP was confirmed by biopsy (transbronchial biopsy, in 3, and open lung biopsy, in 1). Three were smokers, and 1 had a history of occupational exposure to silica. There were no opportunistic infections. Of the 4 patients studied, 3 underwent WLL, showing clinical and radiological improvement, as well as improvement in functional parameters (saturation and DLCO); 1 patient achieved spontaneous remission after quitting smoking. ${ }^{(22)}$

Because of the size of our sample, we cannot accurately determine the impact of GM-CSF replacement therapy or rituximab use on patients with PAP, or their functional follow-up. In this context, the limitations of our study include the fact that secondary treatment options such as GM-CSF replacement therapy and rituximab use were used for a short period of time (because of their limited availability in Brazil), as well as the fact that pulmonary function testing did not include DLCO measurement, together with the fact that serum levels of anti-GM-CSF antibodies were not determined in our sample.

In summary, PAP is a rare and life-threatening disease. To our knowledge, this is the largest case series of patients with PAP ever conducted in Brazil. WLL remains the treatment of choice for symptomatic, hypoxemic patients; however, in some patients, treatment response is unsatisfactory, recurrence is common, or the procedure is contraindicated. For such patients, there are other treatment options, such as GM-CSF replacement therapy and rituximab use, although they are not widely available in Brazil and their roles in such cases have yet to be established. Precautions should be taken in order to avoid complications, especially opportunistic infections, which are common in patients with PAP.

\section{REFERENCES}

1. Borie R, Danel C, Debray MP, Taille C, Dombret MC, Aubier M, et al. Pulmonary alveolar proteinosis. Eur Respir Rev. 2011;20(120):98107. https://doi.org/10.1183/09059180.00001311

2. Baldi BG, Pereira CA, Rubin AS, Santana AN, Costa AN, Carvalho CR, et al. Highlights of the Brazilian Thoracic Association guidelines for interstitial lung diseases. J Bras Pneumol. 2012;38(3):282-91. https:// doi.org/10.1590/S1806-37132012000300002

3. Trapnell BC. Whitsett JA, Nakata K. Pulmonary alveolar proteinosis. N Engl J Med. 2003;349(26):2527-39. https://doi.org/10.1056/ NEJMra023226

4. Jouneau S, Kerjouan M, Briens E, Lenormand JP, Meunier C, Letheulle J, et al. Pulmonary alveolar proteinosis [Article in French]. Rev Mal Respir. 2014;31(10):975-91. https://doi.org/10.1016/j. rmr.2014.08.009

5. Souza RC, Kanaan D, Martins HP, Vianna GA, Amorim VB, Marchiori E. Spontaneous regression of pulmonary alveolar proteinosis: a case report. Radiol Bras. 2012;45(5):294-6. https://doi.org/10.1590/S010039842012000500013

6. Rodríguez Portal JA. Treatment of Adult Primary Alveolar Proteinosis Arch Bronconeumol. 2015;51(7):344-9. https://doi.org/10.1016/j. arbres.2015.02.003

7. Chaulagain $C P$, Pilichowska M, Brinckerhoff $L$, Tabba M, Erban JK. Secondary pulmonary alveolar proteinosis in hematologic malignancies. Hematol Oncol Stem Cell Ther. 2014:7(4):127-35. https://doi.org/10.1016/j.hemonc.2014.09.003

8. Juvet SC, Hwang D, Waddell TK, Downey GP. Rare lung disease II: pulmonary alveolar proteinosis. Can Respir J. 2008;15(4):203-10. https://doi.org/10.1155/2008/528948

9. Leth S, Bendstrup E, Vestergaard H, Hilberg O. Autoimmune pulmonary alveolar proteinosis: treatment options in year 2013 Respirology. 2013;18(1):82-91. https://doi.org/10.1111/j.14401843.2012.02274.x

10. Shah PL, Hansell D, Lawson PR, Reid KB, Morgan C. Pulmonary alveolar proteinosis: clinical aspects and current concepts on pathogenesis. Thorax. 2000;55(1):67-77. https://doi.org/10.1136/ thorax.55.1.67

11. Sawai T, Umeyama Y Yoshioka S, Matsuo N, Suyama N, Kohno S. Autoimmune pulmonary alveolar proteinosis co-existing with breas cancer: a case report. J Med Case Rep. 2014;8:279. https://doi. org/10.1186/1752-1947-8-279

12. Su KC, Lay SL, Perng RP, Chang SC, Chen YM. Lung cancer may develop subsequently or coincidently with pulmonary alveola proteinosis. Lung Cancer. 2007;58(1):144-8. https://doi.org/10.1016/j. lungcan.2007.04.012

13. loachimescu O, Kavuru M. Pulmonary alveolar proteinosis Chron Respir Dis. 2006;3(3):149-59. https://doi. org/10.1191/1479972306cd101rs

14. Pascual J, Gómez Aguinaga MA, Vidal R, Maudes A, Sureda A

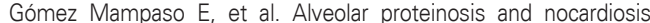
a patient treated by bronchopulmonary lavage. Postgrad Med J. 1989;65(767):674-7. https://doi.org/10.1136/pgmi.65.767.674

15. Pereira-Silva JL, Marinho MM, Veloso TV, Coelho JC JC. Pulmonary alveolar proteinosis and tuberculosis in a diabetic patient: a rare or a seldom diagnosed association? Braz J Infect Dis. 2002;6(4):188-95. https://doi.org/10.1590/S1413-86702002000400006

16. Tekgül S, Bilaceroglu S, Ozkaya S, Coskun A, Komurcuoglu B, Cirak AK. Pulmonary alveolar proteinosis and superinfection with pulmonary tuberculosis in a case. Respir Med Case Rep. 2012;5:258. https://doi.org/10.1016/j.rmedc.2011.11.003

17. Sunderland WA Campbell RA, Edwards MJ. Pulmonary alveolar proteinosis and pulmonary cryptococcosis in an adolescent boy. J Pediatr. 1972;80(3):450-6. https://doi.org/10.1016/S0022 3476(72)80503-

18. Sauni $R$, Järvenpää $R$, livonen $E$, Nevalainen $S$, Uitti J. Pulmonary alveolar proteinosis induced by silica dust? Occup Med (Lond). 2007;57(3):221-4. https://doi.org/10.1093/occmed/kql162

19. Keller CA, Frost A, Cagle PT, Abraham JL. Pulmonary alveola proteinosis in a painter with elevated pulmonary concentrations of titanium. Chest. 1995;108(1):277-80. https://doi.org/10.1378/ chest.108.1.277

20. Patel NM, Diaz-Mendoza J, Valdiviezo EA, Ray C, Simoff MJ. A Case-Series Of pulmonary alveolar proteinosis Treated With Bilateral 
Simultaneous Whole Lung Lavage : A Novel Treatment Modality. Am J Respir Crit Care Med. 2015;191:A4433.

21. Belchior I, Cerdeira AS, Santos M, Braga JS, Aragão I, Martins A Successful pregnancy in a severely hypoxemic patient with pulmonary alveolar proteinosis [Article in Portuguese. Rev Port Pneumol. 2011;17(3):139-42. https://doi.org/10.1016/..rppneu.2011.01.005

22. Lorenzi-Filho G, Carvalho CR, Dohlnikoff $M$, Travassos RR Jr. Proteinose alveolar: apresentação de quatro casos. Rev Hosp Clin Fac Med Sao Paulo. 1991;46(4):196-200.

23. Pereira CA, Sato T, Rodrigues SC. New reference values for forced spirometry in white adults in Brazil. J Bras Pneumol. 2007;33(4):397-
406. https://doi.org/10.1590/S1806-37132007000400008

24. Neder JA, Andreoni S, Castelo-Filho A, Nery LE. Reference values for lung function tests. I. Static volumes. Braz J Med Biol Res. 1999;32(6):703-17. https://doi.org/10.1590/S0100879X1999000600006

25. Thompson JC, Kishima M, Gomes MU, Menezes Mde A, Perandré Neto J, Pereira PT. Pulmonary alveolar proteinosis: four cases. J Bras Pneumol. 2006;32(3):261-6. https://doi.org/10.1590/S180637132006000300013

26. Khan A, Agarwal R. Pulmonary alveolar proteinosis. Respir Care 2011:56(7):1016-28. https://doi.org/10.4187/respcare.01125 